\title{
Jevons et la genèse de la monnaie
}

Une contribution influente malgré de nombreuses apories et un problème de méthode

Pierre Alary

\section{(2) OpenEdition}

\section{Journals}

Édition électronique

URL : http://journals.openedition.org/ei/321

DOI : 10.4000/ei.321

ISSN : 2553-1891

Éditeur

Association Économie et Institutions

Édition imprimée

Date de publication : 30 octobre 2007

Pagination : 155-184

ISSN : 1775-2329

\section{Référence électronique}

Pierre Alary, " Jevons et la genèse de la monnaie », Économie et institutions [En ligne], 10-11 | 2007, mis en ligne le 31 janvier 2013, consulté le 01 mai 2019. URL : http://journals.openedition.org/ei/321 ; DOI : 10.4000/ei.321 
Jevons et la genèse de la monnaie : une contribution influente malgré de nombreuses apories et un problème de méthode

\section{Pierre Alary ${ }^{1}$}

\section{Introduction}

Dans "Money and the mechanism of exchange", Jevons ${ }^{2}$ formalise clairement des principes à l'origine de la genèse de la monnaie. L'absence de double coïncidence des besoins, d'un moyen de mesure homogène de la valeur et d'un moyen de subdivision, limite le niveau des transactions dans une économie de troc. Les agents inventent alors la monnaie pour fluidifier les mécanismes d'échanges. Les travaux de Jevons restent une référence incontournable pour la théorie de la monnaie car ils posent des bases conceptuelles toujours aussi vivantes. La dichotomie économie de troc, économie monétaire, par exemple, est largement reprise dans les recherches contemporaines (BRUNNER, MELTZER, 1971), (STARR, 1972), (WILLIAMSON, WRIGHT, 1994) tout comme la fonction d'intermédiaire d'échange de la monnaie (CLOWER, 1967), (OH, 1989), (OSTROY, STARR, 1990) ou les contraintes de double coïncidence des besoins (KIYOTAKI, WRIGHT, 1989), (KIYOTAKI, WRIGHT, 1991).

Le socle du modèle proposé par Jevons a traversé plus d'un siècle et cela semblerait déjà suffisant pour se pencher sur son travail. Cependant notre intérêt ne s'arrête pas là, il se porte également sur le problème de méthode sous-jacent aux apports théoriques de Jevons. En effet, au-delà des questions spécifiques à l'histoire de la pensée économique, le travail de Jevons mérite une discussion méthodologique dans la mesure où, d'une part la distinction entre approche idéelle ${ }^{3}$ et effective (historique) n'est pas claire et d'autre part cet amalgame est toujours présent dans de nombreux travaux contemporains.

Jevons cherche avant tout des principes séminaux, universels pour expliquer la genèse de la monnaie. Dans ce cadre, il imagine des économies sans monnaie à l'aune des économies marchandes de

1 Enseignant-chercheur à l'Ecole Supérieure de Gestion, Paris.

2 (JEVONS, 1876)

3 Idéelle s'oppose à historique dans ce texte. Une démarche conceptuelle idéelle imagine une économie marchande sans monnaie en retirant la monnaie à ladite économie. Cet état initial n'a peut-être jamais existé, mais il permet de construire un modèle théorique. 
son époque. Dans ces économies imaginaires, idéelles, peu d'échanges s'opèrent mais, conceptuellement, elles fonctionnent sur les mêmes principes que les économies marchandes monétaires. La monnaie n'est qu'un instrument qui autorise un niveau d'échanges supérieur à l'équilibre, un voile jeté sur les phénomènes économiques. En d'autres termes, l'état initial des économies, dans lequel sont identifiés les principes de genèse de la monnaie, est calqué sur un état final auquel la monnaie est retirée. Cette démarche est commune en économie et des auteurs que la période, le cadre théorique et les résultats distinguent nettement, entreprennent le même effort conceptuel : Menger (1892), LordonOrléan (2007). Cependant, contrairement à ces trois auteurs, détachés des phénomènes historiques voire anthropologiques, Jevons voudrait fonder historiquement ses apports valides dans le cadre idéel. Ainsi, la volonté de réconcilier des niveaux d'abstractions différents (idéel et historique) entraîne le problème de méthode évoqué. Problème de méthode d'autant plus aigu que les similitudes entre économies marchandes sans monnaie (imaginées pour comprendre un phénomène) et économies ayant existé dans le passé ne sont pas démontrées dans le texte de Jevons. Selon Polanyi, ce dernier type d'économies serait même très différent des premières : "Par rapport à l'économie antérieure, la transformation qui aboutit à ce système (marchand) est si totale qu'elle ressemble plus à la métamorphose de la chenille qu'à une modification qui pourrait s'exprimer en termes de croissance et de développement continus" (POLANYI, 1996, pg. 70).

Notre travail se penche donc sur le problème de méthode qu'entraîne l'amalgame des deux niveaux d'abstraction. Si la possibilité de confondre les niveaux idéel et historique n'est pas démontrée, ces derniers ne peuvent pas être confondus. Or Jevons ne le démontre pas et discourir sur la démarche qu'il choisit semble important dans la mesure où elle entraîne de nombreuses tensions que son discours souvent contradictoire élude. Sans formaliser clairement ses apories, Jevons avoue la difficulté de comprendre la genèse de la monnaie avec sa méthode : "Currency is to the science of economy what the squaring of the circule is to geometry, or perpetual motion to mechanises" (page VI de la préface). En revanche, que l'amalgame pose des problèmes ne remet pas en cause l'une et l'autre des démarches, elles éclairent différentes facettes de l'analyse économique.

La première partie de ce travail se penche sur l'ouvrage de Jevons. D'une part elle en présente succinctement les résultats. Jevons propose une analyse globale de la monnaie, de sa genèse aux principes de politiques monétaires. D’autre part, elle identifie les 
deux niveaux d'analyse, idéel et historique, présents dans son ouvrage et son désir de rattacher le caractère idéel à des phénomènes historiques.

Les deuxièmes et troisièmes parties se concentrent sur les tensions et sur les problèmes de logique. Les raisonnements sont construits à partir d'exemples de relations bilatérales d'échanges au long cours. Les contraintes ainsi identifiées, issues d'échanges très spécifiques, sont alors généralisées pour traduire une logique endogène. Ce coup de force théorique pose souvent des problèmes et la construction du modèle serait difficile avec des concepts univoques. Dès lors les définitions de la monnaie, du troc et de la mesure de valeur sont polysémiques. Elles restent suffisamment plastiques pour s'adapter aux besoins des démonstrations. Par exemple, les contours de la valeur changent parfois et selon la perspective idéelle ou historique, la valeur entretient des liens plus ou moins étroits avec la monnaie. Enfin, le rôle de l'organisation publique n'est pas clair. L'organisation publique n'intervient pas dans les phénomènes de genèse idéelle, mais elle semble importante lorsque le caractère historique de la genèse est privilégié.

\section{Money and the mechanism of exchange}

\subsection{Une analyse globale de la monnaie}

William Stanley Jevons, 1835-1882, écrit de nombreux ouvrages dont les résultats influencent plus ou moins directement les recherches contemporaines. Tout comme Menger et Walras, Jevons porte la révolution marginaliste. Ces auteurs adoptent une approche ${ }^{4}$ microéconomique et recherchent dans l'action individuelle l'explication de phénomènes généraux. L'ouvrage étudié "money and the mechanism of exchange" (JEVONS, 1876) traduit clairement cette démarche théorique appliquée à la monnaie. Jevons observe des exemples d'échanges bilatéraux ${ }^{5}$ pour isoler des principes théoriques qui expliquent l'apparition d'un instrument, la monnaie, qui facilite les échanges. Ce travail, structuré en 26 chapitres relativement courts, s'intéresse au phénomène monétaire, de la genèse aux formes les plus "élaborées" en 1875. "I have attempted to write a descriptive essay on the past and present money system of the world, the materials employed to make money, the regulations under which the

4 La nomenclature contemporaine et la représentation des espaces microéconomiques et macroéconomiques n'étaient pas encore fixées à la fin du XIXe. Jevons ne bénéficiait pas de ce cadre d'analyse précis et la frontière entre les deux concepts était moins nette. Ainsi à microéconomique nous avons préféré le terme "orientée microéconomie".

5 Par échange nous considérons l'échange de biens ou de services. 
coins are struck and issued, the natural laws which govern their circulation ..." (page V de la préface).

Les trois premiers chapitres constituent le socle de la réflexion. Ils délimitent les principes théoriques nécessaires à la cohérence de l'analyse défendue. Dans le premier chapitre, Jevons se concentre sur la genèse de la monnaie. Pour illustrer sa démonstration, il décrit des relations de troc entre deux agents ou entre deux groupes d'agents et il dégage clairement les contraintes que pose l'absence de monnaie. Jevons repère trois contraintes majeures pour généraliser les échanges de biens sans monnaie : la double coïncidence des besoins, l'absence de mesure de valeur homogène et l'absence de moyen de subdivision.

Le second chapitre joue un rôle singulier et il a une importance capitale. Il pose les bases indispensables à la construction de tous les autres chapitres. Deux parties, intimement liées, directement issues de ses travaux antérieurs (JEVONS, 1957), le structurent. D'une part, la valeur, en opposition aux classiques, est définie. Elle dépend de l'utilité du bien. D'autre part, parce que les biens sont quantifiables en unité quelconque ( $\mathrm{kg}, \mathrm{m}, \mathrm{w}$, etc.) et parce qu'ils possèdent une valeur, les quantités de biens sont comparables et ainsi échangeables. Ce socle bifide assure une cohérence au travail de Jevons : les agents comparent la valeur de biens quantifiables et, pour accroître leur utilité ou leurs profits, ils troquent ces valeurs. Ce deuxième chapitre justifie les mécanismes qui poussent les agents à échanger et il valide en même temps la pertinence des mécanismes présentés dans le premier chapitre. Parce que les agents veulent échanger dans un système peu fluide, ils recherchent un moyen pour faciliter les transactions. La monnaie apparaît alors pour éliminer les frictions immanentes au troc.

Le chapitre III définit les fonctions de la monnaie. Deux fonctions sont jugées fondamentales : l'intermédiaire d'échange et la mesure de valeur homogène (Jevons attribue aux coutumes, aux circonstances, l'élection du support qui remplit ces deux fonctions). La troisième fonction découle directement de la seconde. La monnaie, comme équivalent général, facilite la comparaison des biens quantifiés en unités difficilement comparables au départ $(\mathrm{kg}, \mathrm{m}, \mathrm{w}$, etc.). La quatrième fonction, la réserve de valeur, permet de différer le pouvoir d'achat et elle se retrouve, plus ou moins sous la même forme, comme une fonction contemporaine qui définit la monnaie. En revanche la cinquième fonction se distingue nettement des fonctions contemporaines. Jevons la définit comme l'aptitude à séparer intermédiaire d'échange et réserve de valeur. Pour cet auteur, toutes les fonctions ne se trouvent pas obligatoirement au sein d'un même support monétaire, et la fonction de "séparation" assure cette déconnexion. De plus, cette fonction de "séparation" articule indirectement les trois premiers chapitres conceptuels avec le second 
groupe de chapitres (IV à VII) où l'auteur donne une dimension historique à son travail. Il s'intéresse à l'évolution des monnaies.

Le chapitre IV porte sur les premières formes de monnaies, selon l'auteur, "currency in the hunting State, currency in the pastoral State, currency in the agriculture State" etc.. Ce chapitre a une dimension historique et il présente les premières monnaies dont les fonctions étaient partiellement remplies. Ces monnaies s'apparentent aux "special purpose money" (POLANY, 1968) et, dans cette perspective évolutionniste, la fonction de "séparation" succinctement présentée ci-dessus joue un rôle fondamental. Elle permet de conceptualiser la genèse des monnaies contemporaines "abouties" à partir des "monnaies primitives" aux fonctions dispersées. Comme la monnaie n'apparaît pas ex nihilo, au départ, les quatre premières fonctions sont éparpillées. La cinquième fonction assure une cohérence au modèle évolutionniste où la monnaie regroupe de plus en plus de fonctions.

Le chapitre V se concentre sur les 7 "qualités" nécessaires au support retenu en tant que monnaie. Quatre "qualités" reposent sur les caractéristiques physiques du bien, être facilement transportable (1), indestructible (2), homogène (3), divisible (4). Les trois autres dépendent du rapport que les sociétés entretiennent avec le support: l'utilité et la valeur du bien (5), la stabilité de sa valeur (6), l'aptitude à être accepté comme instrument monétaire (cognizability) (7). Certains métaux regroupent relativement bien ces "qualités" et ils deviennent des monnaies.

Le sixième chapitre présente une série de métaux particulièrement adaptés au monnayage. Le descriptif ne s'appuie pas réellement sur des idées nouvelles contrairement au septième chapitre qui introduit une nouvelle dimension fondamentale dans l'analyse de Jevons. Ce chapitre débute par une série d'exemples de supports monétaires antérieurs à l'apparition des monnaies métalliques. Pour ces premières formes de monnaies, l'auteur évoque l'importance du signe des pièces, les problèmes de titre, de falsification et il introduit un acteur auparavant passé sous silence. L'organisation publique ${ }^{6}$, à travers le Royaume, l'Etat, etc. occupe une place centrale. Elle garantit la validité des pièces et indirectement cela explique la persistance du phénomène monétaire. Ainsi les principes théoriques établis tout au long de ces chapitres ne mobilisent pas la force publique pour expliquer les processus de genèse de la monnaie. Des mécanismes endogènes expliquent ces

6 Selon la démonstration et les exemples qu'il mobilise, Jevons parle de Tribu, de Royaume, d'Empire, d'Etat. Ces appellations semblent traduire la notion de pouvoir légitime, d'entité collective dont l'individu subit les règles. Nous retenons cette idée et nous la qualifierons d'organisation publique pour englober les différents termes retenus par Jevons. 
processus. En revanche, une fois, la monnaie instituée, sa présence repose sur l'autorité publique et cette dernière devient un acteur nécessaire pour garantir la présence de monnaie. L'analyse de Jevons s'oppose à ce titre aux travaux de Knapp. Pour ce dernier, au contraire, la force publique est nécessaire au départ. Ensuite les mécanismes de "bootstrap" expliquent l'évolution du système (KNAPP, 1973).

Les sept premiers chapitres formalisent les mécanismes de genèse de la monnaie et les conditions nécessaires à la pérennité du phénomène. Les autres chapitres la considèrent instituée et se concentrent sur la circulation monétaire, sur les monnaies papier, sur le crédit, sur la masse monétaire optimale, sur la pertinence d'une monnaie internationale, etc. Le travail de Jevons est très éclectique et il embrasse la complexité du phénomène monnaie sans oublier les liens qui l'unissent aux échanges : "the subject of money as a whole is a very extensive one" (page VII de la préface). Cependant, tous les points abordés dans "Money and the mechanism of exchange" ne sont pas traités selon la même intensité et ne possèdent pas la même influence théorique. Seuls les premiers chapitres nous sont plus ou moins directement parvenus et servent de bases aux travaux contemporains qui participent à fonder microéconomiquement la monnaie (ALCHIAN, 1977), (CLOWER, 1995), (JONES, 1976), (OSTROY, STARR, 1974). Ainsi, notre lecture critique portera essentiellement sur les premiers chapitres, les chapitres sur la genèse de la monnaie.

\subsection{Une genèse idéelle et une genèse historique en même temps}

Construire une genèse idéelle semble avant tout le premier objectif de Jevons. Cette démarche éclaire à de nombreux égards sur la monnaie comme le montrent les travaux cités en introduction (Menger et Lordon-Orléan). Ces auteurs, malgré les fortes oppositions théoriques qui les séparent, poursuivent un objectif commun. Ils recherchent des principes absolus, une dimension transhistorique et universelle. Jevons recherche ces principes, mais, en projetant les $a$ priori des économies marchandes sur les économies "antérieures", il tente également de fonder historiquement sa genèse idéelle. Ainsi, à dessein, il mobilise des exemples historiques ou anthropologiques, réels ou fictifs, pour étayer ses propositions théoriques. Sa genèse comporte donc deux niveaux qui se télescopent : un caractère idéel (normatif) et un autre caractère historique. Or ces deux niveaux d'abstraction se distinguent nettement et toute notre critique repose sur leur amalgame. 


\subsubsection{Le caractère idéel de la démarche de Jevons}

À l'aune de ses observations dans des sociétés contemporaines, Jevons semble considérer l'échange avantageux dans tout type d'organisation. Les acteurs sont conscients des avantages que procurent les échanges et, en l'absence de monnaie, ils troquent. Qu'ils n'échangent pas, ou peu, s'explique par des contraintes techniques (l'absence de monnaie ne constitue pas la seule contrainte). Toutes les sociétés seraient animées par cette force interne et l'épanouissement du système est possible une fois les contraintes techniques dépassées. Dès lors, potentiellement, les conditions de l'épanouissement sont toujours présentes et l'échange accroît utilité et profits. Trois présupposés (qu'une approche historique ou anthropologique ne vérifie pas) sont nécessaires au modèle idéel.

- Premier présupposé. Par rapport à l'individu, les échanges permettent d'écouler des biens d'utilité moindre contre des biens d'utilité supérieure. Utilité marginale comme définie dans "The Theory of Political Economy" (JEVONS, 1957). Dans ce contexte, au départ les acteurs troquent pour profiter des avantages liés à l'échange. "exchange has been called the barter of the superfluous for the necessary, and this definition will be correct if we state it as the barter of the comparatively superfluous of the comparatively necessary" (page 8). Ainsi Jevons présuppose qu'à l'origine, universellement, échanger le comparativement superflu contre le comparativement nécessaire assure une satisfaction supérieure que les individus recherchent. Potentiellement, l'organisation sociale permet cette logique.

- Deuxième présupposé. Tout bien est potentiellement échangeable contre n'importe quel autre bien et ainsi la valeur de chaque bien prend corps. Ensuite, toujours potentiellement, un taux d'échange permet de comparer tous les biens pour apprécier les gains que procurent les transferts. "Between one hundred articles there must exist no less than 4950 possible ratios of exchange, and all these ratio must be carefully adjusted so as to be consistent with each other, else the acute trader will be able to profit by buying from some selling other" (page 5).

- Troisième présupposé. Dans un contexte donné il existe un minimum de division sociale du travail en l'absence de monnaie. Chaque acteur ne produit pas la totalité des biens qu'il consomme, les acteurs produisent des biens spécifiques différents et cela entraîne des dotations différentes. Dès lors, du point de vue individuel (de l'unité de production), l'utilité du bien produit en quantité supérieure aux besoins diminue. Ce bien devient alors comparativement superflu contrairement aux biens que l'acteur ne produit pas. De plus, la division sociale du travail rend les échanges 
incontournables dans la mesure où production et consommation sont désynchronisées : "a hunter having returned from a successful chase has plenty of game, and may want arms and ammunition to renew the chase. But whose who have arms may happen to be well supplied whit game, so that no direct exchange is possible" (page 4).

Les quelques exemples retenus ne sont pas exhaustifs et qu'ils soient fictifs ou réels ne change en rien la démarche adoptée par Jevons. Il raisonne avec les a priori d'une économie marchande monétaire qu'il applique à une économie sans monnaie. Il présuppose les principes de fonctionnement énoncés ci-dessus, réels dans l'économie marchande monétaire de la fin du XIXe siècle, universels et l'exemple ci-dessous traduit cette lecture du passé à l'aune du présent "In civilized society the owner of the house may find it unsuitable, and may have his eyes upon an other house exactly fitted to its needs. But even if the owner of the second house wishes to part with it at all, it is exceedingly unlikely that he will exactly reciprocate the feelings of the first owner, and which to barter houses" (page 4). Pour comprendre les contraintes du troc à l'origine, il prend l'exemple du marché de l'immobilier d'une "civilized society" déjà monétisée à laquelle il retire la monnaie. En dehors de la monnaie, les deux types d'économies sont pensées sur les mêmes principes. Cependant, rien ne prouve que les principes soient les mêmes dans une perspective historique. Certains enseignements de l'histoire et de l'anthropologie sembleraient prouver le contraire. En effet, dans les économies "autarciques", tous les biens ne sont pas obligatoirement équivalents entre eux (BOHANNAN, 1959) et la production d'excédent n'est pas obligatoirement recherchée (SAHLINS, 1976). Ainsi, la démarche adoptée par Jevons ne peut pas traduire un phénomène historique même si sa genèse possède explicitement une vocation historique.

\subsubsection{Le caractère historique de la démarche de Jevons}

À l'origine, Jevons voudrait que toutes les économies eussent été des économies marchandes sans monnaie. Ainsi, son travail conceptuel serait historique dans la mesure où le cadre normatif retenu traduirait un phénomène historique. Cette idée sous-jacente à son analyse explique, d'une part le recours permanent à l'histoire et à l'anthropologie et d'autre part, la logique évolutionniste de son travail. Cette logique s'applique au processus d'échange "the earliest form of exchange must have consisted in giving what was not wanted directly for that which was wanted" (page 3), tout comme aux différentes étapes de l'évolution d'une économie : "In modern civilized society the inconveniences of the primitive method of exchange are 
wholly unknown, and might almost seem to be imaginary. Accustomed from our earliest years to the use of money, we are unconscious of the inestimable benefits which it confers upon us; and only when we recur to altogether different states of society can we realize the difficulties which arise in its absence. It is even surprising to be reminded that barter is actually the sole method of commerce among many uncivilized races" (page 2). Historiquement, le troc précède la monnaie quel que soit le type de société. Ainsi, la société britannique de la fin du XIXe ("civilised societies") était précédée par une société de troc qui fonctionnait comme fonctionnent encore les "primitive societies" du XIXe. Dès lors, observer les "primitifs" éclaire sur le passé des "civilisés" et étudier les relations entre "primitifs" et "civilisés" éclaire sur les relations de troc : "There is something absurdly incongruous in the fact that a joint-stock company, called "the african barter company, limited", exists in London, which carries on its transactions upon the West Cost of Africa entirely by bartering European manufactures palm oil..." (page 4). Les exemples choisis dans les récits d'anthropologues permettent donc de comprendre l'apparition de la monnaie dans les "civilised socieites" lorsqu'elles étaient primitives. Pour Jevons, l'exemple anthropologique, réel ou fictif, occupe une place fondamentale. Ils ne sont pas seulement illustratifs. Au contraire, ils servent de point de départ et les observations qu'ils permettent enclenchent la construction théorique. Toutes les contraintes que dégage Jevons proviennent d'un examen attentif des difficultés que rencontrent Melle Zélie et les habitants de la société des Iles, Monsieur Wallace et les habitants d'un archipel Malais ou encore de l'African Barter Company Limited et ses partenaires en Afrique. Jevons construit son raisonnement à partir d'exemples. Contrairement à Menger (1892) il ne théorise pas que dans l'idéel, il veut traduire un phénomène également historique et la cinquième fonction, même si elle peut sembler anodine, joue un rôle essentiel à ce titre (cf. § 1.1). "Separation of functions. It is the highest degree important that the reader should discriminate carefully and constantly between the four functions which money fulfils, at least in modern societies" (page 16). Cette fonction assure, entre autres, la transition des "no proper currency" (postoral, hunting, etc.) vers les monnaies métalliques de l'Europe du XIX" "One of the very earliest mediums of exchange as we have seen consisted of the skins of animals" (page 196). À partir des monnaies observées dans les peuples "exotiques", cette fonction permet d'expliquer l'évolution vers les monnaies abouties, les "all purpose money". Au départ le troc est progressivement remplacé par des "special purpose money", parfois plusieurs monnaies sont nécessaires et progressivement ces monnaies se perfectionnent pour donner des instruments dotés de toutes les autres fonctions. 
Ainsi, quels que soient les usages des évènements historiques et anthropologiques, Jevons veut avant tout établir une genèse conceptuelle de la monnaie. Il tente d'identifier des phénomènes universels, valides quelles que soient les sociétés. Cependant, il voudrait que sa genèse conceptuelle corresponde aussi à l'évolution historique des "civilized socities". Il semble ne pas distinguer la genèse idéelle (établie dans un cadre normatif) et la genèse historique. Or, les deux niveaux d'abstraction sont très différents à en croire les travaux de Polanyi. L'amalgame explique peut-être que Jevons se dispense de vérifier, dans les "incivilized socities", les principes normatifs sous-jacents à la genèse idéelle. En effet, il utilise des concepts polysémiques et un cadre d'analyse imprécis où les deux états sont confondus. Ainsi, selon les besoins de la démonstration, il mobilise des éléments de réflexions issus de l'un ou de l'autre des états. Cette démarche réconcilie en apparence deux niveaux d'abstraction très différents mais cela induit des problèmes d'ordre analytique et logique.

\section{Des exemples choisis à dessein, associés à des concepts polysémiques pour concilier les deux approches}

\subsection{Une genèse conceptuelle établie à partir d'exemples de relations d'échanges très singulières}

L'idée d'une économie marchande sans monnaie ne semble pas correspondre à une réalité historique, ou anthropologique et amalgamer les deux niveaux d'abstraction, que sous-tendent ces méthodologies, entraîne des tensions. Pour les contourner Jevons construit ses propositions théoriques à vocation universelle à partir d'exemples qui ne traduisent en rien un phénomène général. Au contraire, ces exemples sont marginaux, plus ou moins exceptionnels ou spécifiques. En revanche, ils possèdent les caractéristiques recherchées pour démontrer les viscosités liées au troc. Ces exemples semblent "ad hoc" si nous adoptons la définition la plus usuelle de ce terme en économie. Comme les hypothèses du même nom, ils permettent d'obtenir le résultat recherché. Démarche contestée dans "L'ad hoc en économie. La paille et la poutre" (AMABLE, BOYER, LORDON, 1995), dans la mesure où la nature de l'hypothèse transforme le résultat ${ }^{7}$. Jevons recourt à un procédé

\footnotetext{
7 De plus, en dehors des problèmes de méthodologie que pose le recours aux hypothèses ad hoc, ce terme est souvent galvaudé. Les mêmes auteurs contestent le sens du terme ad hoc tel qu'il est utilisé en économie. Si le sens épistémologique était retenu "une hypothèse peut être qualifiée d'ad hoc lorsque son introduction n'accroît pas le degré de réfutabilité de la théorie concernée" (AMABLE, BOYER, LORDON, 1995).
} 
analogue. Il isole certains éléments contextuels (les exemples) pour fonder le principe général à l'origine de la dynamique endogène recherchée. Cette démarche a convaincu et convainc mais les exemples ne sont absolument pas représentatifs d'un phénomène général qui aurait pris naissance au sein même d'une économie sans monnaie.

\subsubsection{Isoler un exemple singulier qui possède les caractéristiques recherchées ou les exemples ad hoc}

Jevons recourt abondamment à l'exemple pour donner un caractère réel et pas seulement normatif à son approche. Il recherche des cas de relations d'échanges où apparaissent clairement les contraintes recherchées et les trois exemples qu'il mobilise dans le premier chapitre : Melle Zélie, Mr Wallace, la Compagnie, même s'ils sont fictifs, traduisent cette assertion.

Les trois exemples reposent sur des relations bilatérales bien spécifiques. Le cas de Mr. Wallace illustre relativement bien le mécanisme mis en valeur dans les deux autres et nous le présenterons brièvement.

Monsieur Wallace, un européen, rentre en contact avec un peuple qui semble vivre en Malaisie. Pour se procurer du poisson Monsieur Wallace propose au poissonnier malais des biens qu'il a emportés (couteaux, etc.). A partir de ce type de relation, entre un européen en voyage et un autochtone, Jevons identifie les frictions immanentes au troc. Jevons s'intéresse à une relation bilatérale particulière mais absolument pas représentative. De plus il ne dit mot ni du contexte malais ni du contexte européen (qui possède déjà la monnaie). La méthodologie qu'il adopte présuppose qu'à l'origine les relations d'échanges internes aux économies aient pu avoir les caractéristiques des relations entre deux économies qui s'ignorent. Si de telles caractéristiques sont semblables, pourquoi les trois exemples portent-ils uniquement sur des échanges entre deux mondes très différents ? Pourquoi ne puise-t-il pas les contraintes endogènes aux sociétés dans la littérature sur les "primitive societies" déjà nombreuse à son époque ? Jevons recourt abondamment aux récits anthropologiques et utiliser les apports de cette science ne semble pas lui poser de problèmes par ailleurs. Ce choix d'exemples de relations entre "primitive societies" et "civilized societies", délibéré semble-t-il, pose deux niveaux de questions dont l'une constitue l'objet du paragraphe 2.1.2.

- D'une part, comme nous le démontrons au $\S 1.2 .2$, Jevons observe les "pimitive societies" de son époque et selon lui les 
"civilized societies" fonctionnaient selon les mêmes principes à l'origine. Ainsi observer les "primitive societies" éclaire sur le passé des "civilized societies" et ce choix méthodologique pourrait se défendre. En revanche, sous couvert d'un tel choix sa démarche emprunte une toute autre voie. En effet, pour découvrir les contraintes à l'origine, Jevons met en relation une société originelle ("primitive societies") avec cette même société une fois développée ("civilized societies"). Dès lors, pour comprendre la genèse de la monnaie, il ne met pas en relation deux "primitive societies" mais une économie à l'état initial avec une économie à l'état final. "Ce choc temporel" met en lumière les contraintes recherchées, mais rien ne prouve que ces contraintes se trouvaient dans les "primitive societies" où le choc ne pouvait pas se produire (les "modern societies" n'existaient alors pas).

- D'autre part, en dehors du "choc temporel" de tels échanges sont caractéristiques de relations au long cours. Ils ne prennent pas corps au sein de chaque économie où les acteurs seraient aiguillonnés par le désir d'échange. Cette seconde remarque rebondit directement sur les critiques adressées par Polanyi aux démarches classiques et néoclassiques en général. Pourquoi choisir des exemples au long cours pour expliquer une logique supposée interne aux économies?

\subsubsection{Les échanges au long cours}

Les caractéristiques des relations de commerce au long cours se trouvent à l'origine du modèle général de Jevons. Cette démarche, généraliser à partir d'exemples particuliers, pose les problèmes méthodologiques que note Polanyi dans "La Grande Transformation" : "le trait de cette doctrine est que le commerce trouve son origine dans une sphère extérieure sans rapports avec l'organisation interne de l'économie" (POLANYI, 1996. pg 90). Selon Polanyi les échanges au long cours ne permettent pas de construire un raisonnement dont la logique émerge au sein des sociétés. Dès lors, la dynamique interne à la société et les contraintes qui auraient pu entraîner la genèse de la monnaie, peuvent-elles être pensées à partir de tels exemples ? Oui, si les échanges au long cours constituent des transactions représentatives entre des agents représentatifs dans une économie marchande sans monnaie. Non, si tel n'est pas le cas. Or, d'un point de vue historique et par rapport aux hypothèses nécessaires à la partie normative du travail de Jevons, le caractère représentatif des relations au long cours semble difficilement défendable.

- Hormis la difficulté de définir un échange représentatif d'un point de vue historique, les échanges au long cours sont spécifiques. 
Ils se distinguent nettement des échanges au sein des économies locales, en revanche ils mettent en relation des économies qui s'ignorent. De plus, ces échanges sont limités, ils concernent quelques biens particuliers dans un contexte, où en amont de la transaction bilatérale sans monnaie, la monnaie existe déjà. En effet, d'après les exemples choisis, la monnaie est présente dans l'une, voire les deux, économies. La "civilized society" qui exporte ses biens en Afrique possède déjà la monnaie et le chapitre V laisse penser que la "primitive society" la possède également (sous une forme plus ou moins aboutie). Ainsi, d'un point de vue historique, les viscosités identifiées par Jevons proviennent de l'absence d'équivalence entre monnaies, appartenant à deux mondes très différents, et les acteurs troquent. Dès lors, ce type d'échange participe assez peu à l'organisation interne de chaque économie. Ils sont spécifiques et le plus souvent liés au pouvoir, au prestige, etc. comme le montrent certains travaux historiques (BOYERXAMBEAU, DELEPLACE, GILLARD, 1986), (BRAUDEL, LABROUSSE, 1977). Ce type d'échanges semble difficilement à l'origine de la dynamique endogène dont parle Jevons.

- Dans une perspective idéelle, il semblerait que les échanges au long cours ne soient également pas représentatifs. Une raison explique la distinction fondamentale entre les échanges internes à une économie et les échanges au long cours. Cette raison se loge au coeur même de la définition de la monnaie proposée par Jevons.

En effet, l'échange au long cours aurait pu être représentatif si Jevons avait défini la monnaie comme un intermédiaire d'échange entre deux individus. Des individus qui s'ignorent troquent dans le cadre de relations bilatérales au départ et ce type de relation présente des contraintes qu'élude la création monétaire. Le troc et la monnaie (intermédiaire d'échange) assurent la même fonction : le transfert de biens. Or la définition de Jevons dépasse largement la fonction d'intermédiaire d'échange et la "measure of value", entre autres, participe à définir la monnaie. Dans ce contexte, il faudrait également que la "measure of value" puisse prendre corps entre deux acteurs qui ne partagent aucune norme. Cette hypothèse ne semble pas se vérifier, au contraire posséder des normes communes constitue un préalable pour qu'apparaisse la "measure of value". Cette fonction s'inscrit dans les représentations collectives (ALARY, 2006), elle constitue le langage (analogie inspirée par: "La violence de la monnaie" (AGLIETTA, ORLEAN, 1982)) et Jevons reconnaît clairement ce caractère. Dans le chapitre III, dans lequel s'amorce la genèse de cette fonction, il souligne l'importance des coutumes et leur caractère premier : "... someone article will usually be selected as money par excellence, by custom or the force of circumstances. This article will begin to be used as a measure of value" (page 13). 
Selon lui, les individus possèdent d'abord une norme culturelle et grâce à ce vécu commun apparaît cette fonction monétaire. Ainsi, si les deux agents qui transactent ne disposent pas de coutumes communes, s'ils ignorent tout l'un de l'autre, le mécanisme de genèse ne s'amorce pas. Dans ce contexte, d'un point de vue idéel, si nous suivons la logique de Jevons (les coutumes constituent le creuset de la fonction : "measure of value"), les échanges au long cours ne peuvent pas servir d'exemple. Le raisonnement de Jevons est contradictoire à ce titre. D'une part, il identifie des contraintes entre des acteurs qui s'ignorent. Les contraintes apparaissent parce que les acteurs s'ignorent et elles entraînent la genèse de la monnaie. D'autre part, des fonctions de la monnaie apparaissent si et seulement si les agents ne s'ignorent pas, si et seulement si ces derniers possèdent des représentations spécifiques à l'organisation interne des économies.

Ainsi, les échanges au long cours ne permettent pas de construire une théorie rigoureuse, que l'approche soit historique ou idéelle. Ces échanges étaient marginaux au XIX ${ }^{\text {e }}$ siècle mais ils possèdent des caractéristiques qui permettent d'identifier les phénomènes recherchés. Cependant, leurs caractéristiques ne se retrouvent pas dans les échanges intracommunautaires et cela explique certainement que Jevons n'ait retenu que des exemples d'échanges au long cours. Ce coup de force théorique réconcilie apparemment genèse idéelle et genèse historique mais il n'est possible qu'avec des concepts flous. Dans "Money and the mechanism of exchange" ces derniers le sont et ils évoluent en fonction des besoins de la démonstration. Si le concept de monnaie avait toujours été défini d'après ses fonctions identifiées dans le troisième chapitre, les échanges au long cours auraient perdu leur caractère opératoire.

\subsection{Des concepts, imprécis, adaptés aux besoins des démonstrations}

Un cadre conceptuel univoque limite les errements théoriques et impose une rigueur analytique. En revanche, des concepts imprécis permettent d'adapter le dit cadre aux besoins de la démonstration. Certains concepts définis par Jevons ne semblent pas toujours précis. Par rapport à nos perspectives de recherche, deux types de concepts sont intéressants à discuter : le troc et la monnaie. 


\subsubsection{Le troc}

"The earliest form of exchange must have consisted in giving what was not wanted directly for that which was wanted. This simple traffic we call barter or truck, the French troc, and distinguish it from sale and purchase in which one of the articles exchanged is intended to be held only for a short time, until it is parted with in a second act of exchange. The object which thus temporarily intervenes in sale and purchase is money." (JEVONS, 1876, pg.3). Cette définition borne relativement bien la notion étudiée et elle offre un cadre général qui isole une relation de troc. Elle précise des notions importantes, id est: le bien recherché doit l'être pour lui-même, "was not wanted directly for that which was wanted" et non pour servir d'intermédiaire d'échange par la suite ; les biens doivent être donnés pour eux-mêmes. Donner ne fait pas référence au don tel que défini par (GODBOUT, 1992), (GODELIER, 1996), (MAUSS, 2002), (SAHLINS, 1976). Pour Jevons, donner a un sens générique et traduit le transfert où n'intervient pas directement la monnaie. Cependant, cette définition ne manque pas d'intérêt et, sous certaines conditions, elle pourrait délimiter le concept de troc. En revanche, ces conditions ne sont pas réunies et le caractère imprécis, par rapport aux objectifs de Jevons, entraîne des problèmes de cohérence.

- Le problème de cohérence. Le problème de cohérence n'apparaît pas au sein de la définition mais entre la définition et la démarche adoptée pour identifier les contraintes spécifiques au troc. Pour identifier ces contraintes, Jevons se concentre sur une relation strictement bilatérale (cf. § 2.1.1). En revanche, la définition du troc dépend aussi des échanges en aval de la relation bilatérale. Si la démarche est purement idéelle, avec une relation bilatérale représentative (entre deux agents représentatifs), alors le modèle est cohérent. Si l'hypothèse théorique d'une relation représentative n'est pas retenue, et les exemples choisis orientent en ce sens, alors le modèle n'est pas cohérent.

Selon les exemples choisis par Jevons pour illustrer ses propositions théoriques, les relations bilatérales ne mettent pas en relation des agents représentatifs. Dans ce contexte, l'étude de l'environnement général est nécessaire pour déterminer si l'objet transféré est "directly wanted". Par exemple, la destination des objets (couteau, poisson) détermine si l'échange procède d'une relation de troc. Soit les objets sont recherchés pour eux-mêmes, ils n'entrent pas dans une nouvelle relation d'échange et alors les acteurs troquent. Soit, l'un des deux objets est recherché pour être échangé par la suite et alors, les acteurs ne troquent pas. Ainsi, 
l'observation d'une relation bilatérale ne suffit pas pour définir le troc. Une relation de troc dépend directement des relations en aval. La relation bilatérale entre A et B est de troc si, et seulement si, ni A ni $B$ ne considèrent les biens échangés comme des éléments qui permettront d'obtenir, grâce à une nouvelle transaction, un autre bien convoité. Quel statut attribuer aux biens échangés par les protagonistes dans la mesure où nous ignorons le dessein des acteurs ? Le poissonnier malais recherche-t-il les biens pour euxmêmes ou pour acquérir d'autres biens ? Si le Malais prend les couteaux de Wallace pour acheter de nouveaux poissons, alors le couteau n'est pas "directly wanted". La relation n'est pas de troc et le couteau "which thus temporarly intervenes in sale and purchase is money". Dans ce cas, Jevons identifie les contraintes supposées au troc, or l'échange est monétaire.

Ce terme "directly wanted" renvoie expressément aux tensions entre les deux approches. D'une part, la dimension idéelle de la démarche de Jevons qui repose sur des relations d'échange représentatives entre des agents représentatifs. Ainsi la relation bilatérale retenue pour identifier les contraintes traduit un phénomène général et il est inutile d'étudier les relations en aval. Par rapport à cette démarche Jevons considère que le poissonnier Malais convoite les biens appartenant à Monsieur wallace pour euxmêmes et que Mr. Wallace désire manger le poisson. D'autre part, la dimension historique laisse penser qu'un bien comme le couteau (ou autre cf. chapitre IV de l'ouvrage) peut circuler. Dès lors, pour déterminer s'il circule, ou au contraire s'il ne circule pas, la compréhension du contexte général est nécessaire. De ce point de vue, l'examen d'une relation bilatérale ne suffit pas pour identifier le troc et il est incohérent de ne regarder les contraintes que dans la relation bilatérale. En effet, Jevons identifie des contraintes supposées spécifiques au troc sans vérifier si la relation est de troc.

- Le problème de précision. La définition du troc mise en relief par les exemples est équivoque et ne règle pas toutes les tensions. Pour délimiter les échanges de troc des échanges monétaires, Jevons introduit une nouvelle notion : "held only for a short time". La notion temporelle permet de distinguer l'économie effective, où chaque agent obtient de l'échange le bien recherché pour l'usage, de l'économie monétaire, où un bien circule pour faciliter ce mécanisme. Dans ce cadre, le bien en question est conservé pour une courte période dans la mesure où il est simplement destiné à obtenir un nouvel objet. Ce niveau d'analyse ne semble considérer que la fonction d'intermédiaire d'échange où le bien (la monnaie) circule entre les acteurs. Cette précision temporelle ne poserait pas de problème si la monnaie n'était qu'un intermédiaire d'échange. Or, elle est aussi une réserve de valeur. Comment réconcilier "held only 
for a short time" et la réserve de valeur ? La fonction de réserve de valeur s'oppose précisément à la circulation (ORLEAN, 1991), or conserver la monnaie sur une longue période ne rend pas caduque son statut de monnaie. La notion temporelle semble poser de gros problèmes pour distinguer une relation de troc d'une relation monétaire. Si la courte période est définie inférieure à $n$, toute monnaie thésaurisée sur une période supérieure à $\mathrm{n}$ n'est plus une monnaie. Que devient-elle ? Un élément "non désiré directement" ? Dans ce cas, si la courte période est supérieure à n, retombons-nous dans une relation de troc?

Par ailleurs, lorsque Jevons observe l'économie contemporaine de son pays, il observe un troc qu'il semble distinguer du troc "originel" : "the farm labourer may partially receive payment in cider, or barley, or of a piece of land. It is always been usal for the miller to be paid by a portion of the corn which he grinds" (page 6). Cependant, "In almost all acts of exchange money intervenes in one way or other, and even when it does not pass from hand to hand, it serves as the measure by which the amounts given and received are estimated" (page 5). Dans les économies contemporaines, les acteurs troquent et l'unité de compte monétaire fluidifie leurs échanges. En revanche "Commerce begins with barter and in certain sense it returns to barter; but the last form of barter, as we shall see, is very different from the first form" (page 5). Les deux trocs sont radicalement différents selon Jevons mais, en même temps, la définition du troc initial ne permet pas de le distinguer, conceptuellement, des autres formes trocs (troc 2). Les exemples, imaginés pour mettre en relief des contraintes, ne peuvent pas être confrontés à un cadre rigoureux. Dès lors, il est demandé de croire que le troc de gibier entre "primitive", ou le troc de Monsieur Wallace, possèdent les caractéristiques du troc "originel" et non les caractéristiques du troc de services de mouture contre du grain. Nous devons accepter que les viscosités identifiées grâce à l'exemple de Melle Zélie et des habitants de "Society of Islands" (page 1) prennent corps en dehors de toute unité monétaire (idem pour Monsieur Wallace). Nous devons accepter que les contraintes sont spécifiques au troc "originel" même si par ailleurs Jevons se réfère aux prix des biens sur le marché des Halles à Paris pour évaluer le montant de la rémunération de Melle Zélie. En effet, il est nécessaire que les types de relations d'échanges, où apparaissent les contraintes identifiées par Jevons, soient indépendants d'une unité de compte monétaire. Sinon, la genèse de Jevons repose sur des contraintes de troc 2 dans un contexte où la monnaie existe déjà. Or, ce troc ne peut pas servir à comprendre la genèse selon Jevons.

Jevons propose une définition générique du troc, suffisante dans un contexte général mais insuffisante pour construire une 
genèse à partir du troc. Le concept de troc est trop vague pour une telle genèse dans la mesure où il est défini par défaut. En effet, pour cette définition, le concept de monnaie est nécessaire. Elle renvoie dos-à-dos troc et monnaie et elle ne permet pas de confronter les exemples, destinés à identifier des contraintes, à un cadre rigoureux. En revanche, elle permet un va et vient plus ou moins élégant entre approche idéelle et historique. Ce va et vient tente de réconcilier les approches mais il montre surtout les difficultés que rencontre un travail de définition du troc. Chapman disait : "But barter is like a small fish, difficult to grasp and almost impossible to hook." (CHAPMAN, 1980, pg. 74)

\subsubsection{La monnaie ou les monnaies}

Le caractère imprécis du concept de troc se retrouve également dans la définition de la monnaie. Dans le premier chapitre, Jevons semble assimiler le troc à toute forme d'échange bilatéral n'ayant pas recours physiquement à une monnaie métallique. Il qualifie alors les économies où s'effectuent de tels échanges, d'économies "where there was no proper currency". Il emploie "currency" et non "money" si l'instrument ne ressemble pas à une monnaie métallique et cela rebondit sur un double problème : de terminologie et analytique.

Au départ, Jevons distingue sans précision formelle "currency" et "money". Ensuite, paradoxalement, dans le chapitre "Early History of Money" il semblerait que les deux termes, currency et money, soient indifféremment employés. Les "currencies" ne sont pas toujours des monnaies à part entière. L'utilisation imprécise du terme perturbe parfois la compréhension des mécanismes et si "currency" représente la monnaie, pourquoi entretenir l'ambiguïté ?

En revanche si "currency" égale monnaie "incomplète", alors pourquoi "no proper currency" ? Y a-t-il une différence entre "currency" (monnaie "incomplète") et "no proper currency" ? Toujours selon la même logique, que signifie alors une "proper currency", est-ce une monnaie?

Sans trouver de réponse dans l'ouvrage de Jevons, nous nous interrogeons sur les différences entre : "money, proper currency, currency et no proper currency". L'utilisation imprécise de ces termes ne poserait pas de problèmes si Jevons adoptait une nomenclature avec d'un côté le troc (clairement défini) et d'un autre côté la monnaie selon ses différentes formes (des plus "primitives" aux plus "abouties").

Or tel n'est pas le cas. Parfois, les "no proper currency" se retrouvent dans un "state barter" et parfois, lorsqu'elles sont définies par leurs fonctions, elles sont des monnaies. En effet, il retient des peaux comme monnaie "even after the use of coin had become 
common among the indians the skin commonly used as the money of account" (page 21) ou encore, il admet la possibilité qu'une monnaie en bois ait pu exister (page 29). Si les "no proper currencies" appartiennent à un "state barter" alors sont-elles des biens troqués même si elles possèdent certaines fonctions de la monnaie? Si oui, pourquoi qualifier par ailleurs des peaux "as the money" ? Si les fonctions definissent la monnaie ("the several functions of money were thus clearly performed by different commodities at this early period" (page 21)), pourquoi une "no proper currency" qui possède des fonctions (unité de compte par exemple) appartient-elle à un state barter?

Nous ne disposons d'aucun élément pour différencier clairement certaines monnaies du troc au départ et cela pose clairement un problème pour comprendre les principes de genèse. Le concept de monnaie défini par les fonctions est difficilement applicable aux exemples (pour identifier les monnaies par exemples). Dès lors, selon les cas, déterminer les critères qu'applique Jevons pour distinguer troc et monnaie est complexe et les pièces métalliques sont le seul élément clairement identifié. Elles sont des monnaies et assez souvent, mais pas toujours, il réduit la monnaie aux pièces métalliques de son époque.

\section{Les imprécisions conceptuelles rebondissent sur deux problèmes spécifiques à la théorie de la monnaie}

\subsection{La commensurabilité : un problème majeur}

Si notre interprétation des premiers chapitres du travail de Jevons est correcte, les biens ont une valeur comparable, et les acteurs déterminent ainsi l'avantage procuré par l'échange. Cependant, comparer la valeur des biens est difficile, cela pose un problème ordinal et cardinal, et pour faciliter le processus de comparaison, la monnaie est inventée. Les fonctions de mesure de la valeur et d'unité de compte assurent la commensurabilité ${ }^{8}$. Pour certains auteurs (AGLIETTA, ORLEAN, 1982), (ORLEAN, 2003), d'un point de vue strictement idéel, cette démarche ${ }^{9}$ pose déjà des problèmes qui

8 A ce titre, la mesure de valeur et l'unité de compte, présentées pages 13 et
14 , constituent deux fonctions de la monnaie. Jevons leur attribue une place
singulière et distincte, elles sont deux, en revanche, les distinguer
conceptuellement n'est pas toujours facile. Il semble que sous ces deux
fonctions la même notion soit plus ou moins traduite.
9 L'économie marchande de troc permet à la valeur marginale de prendre
corps, la valeur permet la comparaison et le désir d'échange peut s'exprimer, 
prennent de l'ampleur lorsque l'analyse tente de retracer en plus des phénomènes historiques. Un premier niveau de problèmes touche le coeur de la logique du modèle de Jevons. Sans une mesure de la valeur les mécanismes de l'échange semblent difficilement amorçables. Or, ces mécanismes sont supposés entraîner le process de genèse des deux fonctions précitées. Le second niveau de problèmes découle du premier. Jevons cherche des subterfuges pour masquer les contradictions immanentes à son modèle.

Les premiers chapitres de Jevons cherchent des principes premiers pour expliquer la genèse de la monnaie et une lecture attentive ne permet pas réellement de dégager le principe premier. D'une part, les biens ont une valeur. Parce qu'ils ont cette valeur des échanges s'opèrent (troc) et progressivement la monnaie est créée. La valeur est le principe premier, elle constitue le point d'encrage nécessaire au positionnement des acteurs pour déterminer leurs choix d'échanges. D'autre part, la valeur n'est pas intrinsèque "utility and value are not intrinsic..." (page 9). Elle prend corps par le système d'échanges basé sur le troc. Pour ce second aspect, le système d'échanges est le principe premier.

Dès lors, quel est le principe premier, il ne nous apparaît pas clairement dans l'ouvrage de Jevons et cela a un impact direct sur la logique de genèse. En effet, si la valeur est le principe premier, elle n'est pas marginale. Si le système d'échange est le principe premier, comment s'enclenche-t-il sans la valeur au préalable? La nomenclature des biens (BENETTI, CARTELIER, 1987) règle apparemment ce problème mais une telle hypothèse n'est absolument pas satisfaisante lorsque l'auteur s'écarte d'un modèle strictement idéel. Jevons s'en écarte et les prolongements historiques de son travail semblent contredire en même temps les deux analyses ci-dessus. En effet, ni la valeur ni le système d'échange ne sont des principes premiers dans la mesure où la monnaie, parce qu'elle permet la comparaison cardinale et ordinale, est ce principe. La monnaie donne corps à la valeur et ainsi, le système d'échange (le rapport marchand) peut s'enclencher comme le montrent plusieurs exemples.

Premier exemple: Nous évoquons l'importance des coutumes dans le paragraphe 2.1.2. consacré aux échanges au long cours. Les coutumes, les représentations communes semblent nécessaires selon Jevons pour offrir à la "measure of value" (l'unité de valeur, la représentation homogène de la valeur), les conditions de sa genèse. Dès lors, cette partie du discours de Jevons retient la "measure of value" comme une construction sociale, une perception commune

l'absence d'une "mesure of value" complique les transactions et alors la monnaie est inventée 
(un langage) pour communiquer sur les biens. La "measure of value" peut donc exister indépendamment du système d'échange. Rappelons par ailleurs que la séparation entre fonctions constitue la cinquième fonction de la monnaie selon Jevons (§ 1.1). Dans ce contexte grâce à la fonction de "séparation", une seule fonction permet de définir la monnaie. Ainsi, qu'il y ait échange ou pas, si la coutume attribue la fonction de "measure of value" à un support, il est monnaie. Indirectement, par ses observations anthropologiques, Jevons dit que la monnaie (measure of value) précède le rapport marchand, qu'elle en est même une condition. Fonder historiquement des propositions théoriques idéelles pose à Jevons des problèmes de logique. Lorsqu'il analyse les économies ayant existé, il semble donner raison aux recherches d'Aglietta-Orléan.

Deuxième exemple: Comme le montrent les exemples choisis par Jevons, les relations de $\operatorname{troc}^{10}$ ne permettent pas aux acteurs d'échanger ou marginalement (en dehors des européens nous ne savons pas si les autres désirent échanger). En effet, le troc ne permet pas l'amorce du mécanisme d'échange et monsieur Wallace n'arrivait même pas à se procurer sa nourriture en troquant : "When Mr. Wallace traveliing in the Malay Archipelag, he seems to have suffered rather from the scarcity than the superabundance of provisions" (Jevons, 1876, p. 2). Sans la représentation commune (measure of value) la relation bilatérale de troc était pratiquement impossible dans la mesure où de longues négociations étaient un préalable à tout échange: "In this most interseting account of his travels, he tells us that in some of the islands, where there was no proper currency, he could not procure supplies for dinner with-out a special bargain and much chaffering upon each occasion" (page 2). Ces négociations ${ }^{11}$ étaient nécessaires pour étalonner les différentes

10 Jevons n'attribue pas l'absence d'échange à des problèmes de transports ou autres.

11 Barter provient du vieux français barater (WILLIAMSON, 1994). Ce terme signifie tromper (DICTIONNAIRE) "Barat, m. acut. Est tromperie, fraude, principalement en marchandise, Fraus, dolus malus, deceptio. Ainsi l'on dit, Contracter sans fraude, barat ne malengin, Bene pacisci ac sine fraudatione, Bona fide conuenire. C'est un mot grandement usité és pays de Languedoc, Provence, et adjacents. Lesquels en font un verbe actif en leur langue, Barator, c'est Barater, qui signifie tromper autruy en fait mesmement de marchandise, vendant, acheptant ou trocquant, et en usent aussi pour trocquer ou eschanger une chose à autre. Et outre encores en font un nom adjectif, Baratier, et Baratiere, pour celuy ou celle qui est coustumier de frauder autruy, Fraudulentus, Fraudator, Fraudulenta, Fraudatrix. \Barater, verb. act. acut. Decevoir, tromper, voyez Barat. \ Barateur, m. acut. Decepteur, trompeur, frauduleur, voyez Barat".

Baratiner en français usuel provient également du vieux français barater. 
représentations selon une base commune nécessaire à l'échange. Sans cette perception les acteurs négociaient pour déterminer "at what rate any exchange to be made" (page 5) et cela permet quelques échanges occasionnels et irréguliers comme nous venons de le voir. Les "price takers" ne peuvent pas se positionner par rapport à des taux d'échanges connus de tous dans la mesure où, faute d'une représentation commune pour la concevoir, les taux n'existent pas. Cette absence de taux entre les protagonistes explique certainement la pirouette de Jevons. Pour démonter l'intérêt d'une relation bilatérale de troc et la logique endogène sous-jacente qui entraîne la genèse de la monnaie, il prend des prix, des taux d'échanges, extérieurs à la relation étudiée. Les gains que Melle Zélie réalise avec les tribus lointaines, dans des pays lointains, sont callés sur la grille de prix en vigueur au marché des Halles à Paris " in the halle in Paris, as the primer donna remarks in her lively letter, printed by Mr. Wolowski, this amount of live stock and vegetable may have brought for thousand francs, which should have give a good remuneration for five songs" (page 1). La grille est complètement extérieure à la relation de troc entre les protagonistes. En revanche, elle constitue la cheville ouvrière du système d'échange. Elle joue le rôle du journal qui publierait régulièrement les prix des biens (page 26) ou bien celui du commissaire-priseur, extérieur à la logique endogène, mais nécessaire au modèle Walrassien.

Dès lors, si l'organisation marchande et la logique endogène ne sont pas possibles sans des références, des taux d'échanges, exogènes au départ, comment les moyens de comparer les biens sans monnaie étaient-ils présents dans une économie originelle purement endogène ? De plus dans cette économie originelle (sans référence exogène), si l'organisation marchande n'est pas possible sans monnaie, comment des biens peuvent-ils circuler entre les acteurs pour que l'un deux soit élu comme la monnaie ? "chosen commodity becomes a common denominator or common measure of value in terms of which we estimate the values of all other goods, so that their values become capable of the most easy comparison" (page 5). Ces questions restent en suspens tout comme les réponses qui permettraient de clarifier le rôle de l'organisation publique.

\subsection{L'importance de l'organisation publique et son rôle ambigu}

L'organisation publique ${ }^{12}$ semble occuper une place déterminante. De nombreuses allusions émaillent le discours de Jevons et, à partir d'observations, il formalise clairement cette importance dans les chapitres VII, VIII, XVI. Ces chapitres

12 Cf. définition en note de bas de page $n^{\circ} 4$. 
superposent au modèle initial le rôle de l'organisation publique pour garantir au phénomène monétaire les conditions de sa durabilité. En revanche, dans le modèle initiale (les processus de genèse de la monnaie (chapitre I, II, III)) l'organisation publique n'intervient pas et déterminer le moment où elle devient nécessaire est difficile.

- Une fois la monnaie apparue de façon endogène, le phénomène monétaire ne serait-il pas durable sans l'organisation publique ?

- Ou bien, une fois la forme "primitive" de monnaie apparue, sans l'organisation publique, les mécanismes de genèse de la monnaie ne pourraient-ils pas évoluer vers une monnaie "aboutie" ? Si tel est le cas, soit l'organisation publique est nécessaire à la genèse, soit les monnaies "primitives" ne précèdent pas la monnaie "aboutie".

Introduire le rôle de l'organisation publique participe à la logique suivie par Jevons à certains égards mais cela entraîne un lot supplémentaire de tensions. En effet, il ne dégage pas nettement l'antériorité de la force endogène par rapport au rôle de l'organisation publique. Ce point complète et rebondit sur le $\S 2.1$.2. où la coutume est antérieure à la logique endogène pour expliquer la mise en place d'une norme reconnue par les coéchangistes (la fonction de "measure of value").

\subsubsection{Pourquoi l'organisation publique est-elle nécessaire selon Jevons?}

Selon Jevons, la monnaie se déprécie et ce phénomène général de dépréciation commence sitôt la monnaie apparue : "thus there is a natural tendency to the depreciation of the metallic currency, which can only be prevented by the constant supervision of the state" (page 82). Deux phénomènes expliquent la nécessité de l'organisation publique pour donner à la monnaie un caractère durable. D'une part, sans l'organisation publique pour garantir le titre des pièces, et le faire respecter, les agents fraudent. La fraude altère la confiance des agents et ils rejettent alors la monnaie. Jevons prend de nombreux exemples pages 60 et 61 pour illustrer l'importance des dessins sur la tranche des pièces par exemple. Lorsque le métal est retiré ils disparaissent et le prélèvement illicite est rendu visible : "... which the weight and fineness are certified by the integrity of designs ..." (page 57). Dans ce contexte, parce que l'organisation publique propose une monnaie clairement identifiée (dont la "valeur" est reconnue) et parce qu'elle punit les contrefaçons, les acteurs ont confiance en la monnaie et l'utilisent. D'autre part, Jevons, s'appuyant sur la loi de Gresham (page 82), distingue clairement la monnaie d'un objet quelconque. Un objet quelconque est recherché pour lui même. Les acteurs, selon leurs critères, recherchent donc le meilleur d'entre eux et les entreprises sont 
incitées à produire les meilleurs objets. Un mécanisme inverse guide le rapport à la monnaie. La monnaie est faite pour circuler et chaque acteur recherche la monnaie dans la mesure où les autres l'acceptent. Sous cette condition, même la pire des monnaies, lorsqu'elle permet de bons profits, est recherchée. Seule l'intervention de l'Etat peut enrayer ce mécanisme pour conserver la "bonne" monnaie. Ainsi, des forces endogènes entraînent la genèse mais ce processus de genèse ne serait pas durable sans le pouvoir supra individuel de l'organisation collective. Considérée sous cet angle, l'analyse de Jevons semble réconcilier approche idéelle et historique, mais son argumentaire ne suit pas toujours cette ligne directrice. Il pose réellement le problème du rôle de l'organisation publique au sein même de la genèse de la monnaie.

\subsubsection{Pourquoi la postériorité de l'organisation publique n'est- elle pas réellement dégagée?}

L'importance de l'organisation publique ne s'oppose pas réellement au caractère idéel du modèle proposé par Jevons. Des forces endogènes poussent les acteurs à créer la monnaie pour faciliter les échanges et, une fois la monnaie créée, pour les raisons présentées ci-dessus, l'organisation publique est nécessaire. L'intervention publique "stabilise" le système monétaire et ainsi il devient durable. Cette méthode permet d'intégrer au modèle idéel l'organisation publique, dont l'influence transparaît clairement dans les exemples qu'il mobilise, mais elle pose un problème de logique. En effet, comme nous le disions, l'antériorité des forces endogènes, clairement établie dans les premiers chapitres, ne peut pas être vérifiée par la suite. Deux cas de figure se présentent.

- Les fonctions définissent la monnaie et les monnaies "primitive" ne s'inscrivent pas obligatoirement dans le processus de genèse de la monnaie "moderne" (monnaie métallique des pays industrialisés à la fin du XIX ${ }^{\mathrm{e}}$ siècle, cf.2.2.2). Les différents types de monnaies ne peuvent prétendre à l'universalité (SERVET, THERET, YILDIRIM, 2007) et une fois instituées leur pérennité dépend de l'organisation publique. Cette option aurait une cohérence, si Jevons définissait réellement la monnaie par les cinq fonctions ( $\$ 1.1$ ) pour déterminer le point à partir duquel un "objet" est institué en tant que monnaie. Or tel n'est pas le cas (§ 2.2.2), il est impossible de déterminer ce point et ainsi les liens ou l'absence de liens entre les différents types de monnaies.

- Jevons semble au contraire attribuer un caractère universel à la monnaie des pays industrialisés. Il retient toujours des exemples dans les sociétés industrielles, lorsqu'il parle d'un phénomène abouti. Les monnaies "primitive" participent à un processus 
évolutionniste plus ou moins long comme le prouvent les qualificatifs qu'il emploie (§ 2.2.2). Deux cas de figures apparaissent également à ce niveau :

- Soit les monnaies "primitive" ne sont pas réellement des monnaies et elles constituent des éléments de la genèse de la monnaie. Dans ce cas, si la genèse est endogène l'organisation publique ne doit pas intervenir, sinon la genèse n'est pas qu'endogène. En revanche, si les étapes (monnaies "primitive") ont besoin de l'organisation publique pour ne pas mourir avant d'évoluer en monnaie, alors l'organisation publique est un élément nécessaire au processus de genèse. Dans ce contexte, pour vérifier ces deux hypothèses la question suivante se pose : Est-il envisageable que les étapes (les monnaies "primitive") ne soient pas soumises aux mêmes contraintes que la monnaie? Comme les monnaies, les étapes (monnaies "primitive") circulent et tout acteur aura intérêt à rechercher la pire des étapes acceptées par autrui, voire les falsifier. De plus, si les monnaies "primitive" sont des étapes de la genèse, dans quelles mesures peut-on les produire avec les lois ordinaires de la concurrence ? Un cauri qui passe de main en main comme une monnaie primitive, peut être produit comme un bouton et la remarque de Jevons pourrait s'appliquer au cauri : "can we trust to the ordinary competition of manufacturers and traders to keep up a sufficient supply of such coins (les bonnes pièces) just as they supply buttons or pins and needles ? Or must we establish a government department under strict legislative control to secure good coinage?" (page 64). Ainsi, l'organisation publique est également nécessaire pour assurer le contrôle de la fabrication des cauris, elle devient alors un élément de la genèse de la monnaie moderne et elle doit être intégrée au modèle en tant que telle. En effet, les étapes (monnaie "primitive") ont besoin de l'organisation publique pour ne pas mourir avant d'évoluer en monnaie, alors l'organisation publique est un élément du processus de genèse.

○ Soit les monnaies "primitive" sont des monnaies à part entière, elles suivent leur propre chemin et elles ne sont pas obligatoirement des étapes de la genèse de la monnaie. Ce niveau d'analyse repose sur la monnaie avec ses différents types ("primitive", modernes) (SERVET, THERET, YILDIRIM, 2007). En revanche, pour de multiples raisons, même si les différents types de monnaies sont des monnaies à part entière, rien n'interdit de penser que les monnaies "primitives" aient permis l'apparition des monnaies modernes. Dans ce contexte, si les monnaies "primitive" ont pu intervenir dans la mise en place de la monnaie moderne, alors, comme l'organisation publique a assuré la "survie" des monnaies "primitive", elle est nécessaire à la mise en 
place de la monnaie moderne. En effet, sans l'organisation publique, pour les raisons évoquées ci-dessus, les monnaies "primitive" auraient disparu. Ainsi, la genèse de la monnaie ne fait pas appel à l'organisation publique, mais la monnaie moderne, un type de monnaie particulière - la monnaie moderne permet l'organisation marchande de la fin du XIX : "Modern society could not exist in its present complex form without the means which money constitutes of valuing, distributing and contracting for commodities of various kinds" (page 3) - ne serait jamais apparue sans l'organisation publique.

Un troisième niveau, même s'il ne participe pas directement à la démonstration, traduit l'ambivalence de Jevons quant au rôle de l'organisation publique. Lorsque Jevons observe des monnaies existantes ou ayant existé il leur attribue un caractère hautement symbolique : "Some of these coins are at once treasured up in cabinets in the manner of medals" (page 63). Il décrit comment le pouvoir (l'organisation publique) utilise la monnaie pour transmettre une image: "the German states, especially Prussia, have struck a long series of beautiful coins ..." (page 63). Les monnaies permettent également de retracer l'histoire des dynasties : "... and the history of the kings of Prussia would be traced out by future numismatists ..." (page 63). Pour Jevons les monnaies sont donc inféodées au pouvoir légitime, elles symbolisent le pouvoir et, en même temps, le pouvoir pérennise le phénomène monétaire.

Le rôle de l'organisation publique montre certaines imprécisions. L'organisation publique n'intervient pas dans le processus de genèse, il est endogène, mais l'absence de concept monosémique de monnaie, la présence de plusieurs formes de monnaie (dont les liens ne sont pas réellement spécifiés), la perspective évolutionniste, ne permettent pas de déterminer à quel moment elle intervient. Dès lors, selon la façon dont l'ouvrage est lu, l'organisation publique peut intervenir après la genèse, pendant la genèse ou en être le point de départ. La "measure of value" (§ 2.1.2) a besoin des coutumes au sein d'une communauté pour prendre corps comme le montrent certains aspects de son analyse.

\section{Conclusion}

Dans son ouvrage, Jevons propose avant tout une genèse conceptuelle de la monnaie. Il veut mettre en évidence des principes séminaux, universels, à partir d'un cadre normatif. Cependant, il voudrait également démontrer que ce cadre correspond à une réalité 
historique et/ou anthropologique. Pour cela il assimile les économies anciennes (originelles) et les économies qui échangent peu ("primitive"), à des économies contemporaines sans monnaie. Comme ces dernières, les premières sont animées par une force basée sur le désir d'échange des individus, mais les frictions liées au troc limitent le niveau des échanges. La genèse de la monnaie constitue une réponse aux frictions, elle fluidifie les mécanismes d'échanges. Cependant, ce modèle repose sur une hypothèse forte : les deux économies sont similaires. Polanyi réfute cette hypothèse et il semblerait que l'étude des économies existantes, ou ayant existé, lui donne raison. Dès lors l'analyse de Jevons rencontre de nombreuses tensions pour réconcilier les deux niveaux d'abstraction et un certain nombre d'imprécisions, voire de contradictions, émaillent son discours. Premièrement, il donne un caractère historique à son analyse idéelle en recherchant des exemples singuliers pour isoler le phénomène recherché. De plus, l'exemple est souvent extrait de son contexte et, resitué, le phénomène identifié ne serait plus valide. Ensuite l'exemple particulier, partiel et marginal, est généralisé pour traduire un principe général. Deuxièmement, les concepts essentiels à l'analyse sont équivoques. Ces derniers restent imprécis et ils s'adaptent aux besoins des différentes démonstrations. Le concept de monnaie par exemple est clairement défini par cinq fonctions d'importance inégale dans les premiers chapitres. Lors des démonstrations, Jevons ne vérifie jamais si le support retenu possède les caractéristiques identifiées par le concept. Troisièmement, les principes premiers à partir desquels le modèle est construit sont différents selon la perspective défendue par l'auteur. Dans certains cas, la valeur est un principe premier. Les individus comparent la valeur des biens et ainsi les échanges se réalisent. L'absence d'un moyen pour comparer la valeur selon une norme homogène limite le niveau des échanges et alors la monnaie est créée pour remplir cette fonction d'évaluation. En revanche, la fonction d'évaluation est permise grâce aux coutumes, à des représentations communes aux individus. Cette fonction existe donc déjà et il est difficile de comprendre pourquoi elle serait créée pour faciliter les échanges. Cette fonction, parce que Jevons choisit des exemples qui mettent en relation des peuples qui s'ignorent, est uniquement absente des exemples choisis. Par ailleurs, lorsque la partie historique domine son discours, Jevons reconnaît la quasiimpossibilité d'échanger sans cette norme commune. Il admet alors que la monnaie est indispensable au mode d'organisation marchand contrairement aux parties idéelles où il postule que la monnaie naît de l'ordre marchand. Enfin, le rôle de l'organisation publique n'est pas facile à cerner, elle ne devient nécessaire qu'une fois la monnaie instituée. En revanche, comme la monnaie n'est pas clairement définie, entre le troc et les monnaies des économies industrielles de 
la fin du XIXe, il est difficile de déterminer à quel moment elle devient nécessaire.

Cependant, quelles que soient nos critiques l'analyse de Jevons ne manque pas d'intérêt. Elle pose des éléments de réflexion à partir desquels une analyse critique, ou complémentaire, peut approfondir des principes de genèse. Elle montre les limites d'un travail qui repose sur des bases conceptuelles équivoques. Il n'existe pas de cadre suffisamment stable pour étayer un raisonnement robuste et elle met en exergue les problèmes que rencontre la confusion entre genèse idéelle et historique. Ces deux niveaux d'abstractions ne peuvent être réunis. Elle montre également les limites de la genèse idéelle. Cette dernière imagine des principes de genèse à partir d'un état final. Or si l'état final, de l'état de nature (sans monnaie), n'a jamais existé cette démarche n'explique pas la genèse de la monnaie en tant que telle. Certes, elle met en avant le rôle de la monnaie : son caractère "constitutif" pour une économie marchande ; le vide créé par l'absence d'unité de compte par exemple, mais elle n'explique pas la genèse de la monnaie. Ces deux aspects sont très différents comme le montre l'exemple trivial suivant. Imaginons un cadre idéel : la roue a été inventée pour limiter les frottements lors des déplacements d'une voiture. Nous retirons la roue à une voiture pour comprendre la genèse de la roue. Nous concluons : soit la roue était une condition préalable à la fabrication de la voiture, soit la roue est un instrument qui facilite le déplacement de la voiture. Même si nous concluons que la roue est une condition préalable à la fabrication de l'auto, nous expliquons les conditions d'existence de la voiture mais pas la genèse de la roue. Historiquement la roue semble avoir été faite pour battre le grain, elle a évolué et a permis la construction des automobiles et, sous une autre forme, elle permettra peut-être la création d'un outil dont nous ignorons tout aujourd'hui. Ainsi, pour comprendre la genèse de la monnaie, et non les conditions d'existence d'une économie marchande, la genèse historique nous semble la seule approche et nous avançons l'hypothèse suivante (hypothèse en tant que conjecture et non postulat (ad hoc)) : le langage que constitue la monnaie est apparu dans les sociétés lointaines et il était un élément essentiel à la construction de liens sociaux entre des individus. En tant que représentation sociale il permettait de quantifier les actes sociaux, le rapport des individus à leur communauté, la comparaison de certains biens, etc. Ces actes étaient essentiels et, progressivement, selon des règles parfois complexes, de plus en plus de biens sont devenus échangeables parce que comparables. Dans ce contexte, la norme donne corps à la valeur d'un nombre croissant de biens et l'économie marchande se met en place. 


\section{Bibliographie}

AGLIETTA, M., ORLEAN, A., (1982). La Violence de la Monnaie. Paris, Presses Universitaires de France.

ALARY, P. (2006). "Dynamique monétaire et développement des échanges marchands". Secondary "Dynamique monétaire et développement des échanges marchands". Paris, Ecole des hautes Etudes en Sciences Sociales: 652.

ALCHIAN, A. (1977). "Why Money ?" Journal of Money, Credit and Banking 9 (1): 133-140.

AMABLE, A., BOYER, R., LORDON, F., (1995). "L'ad hoc en économie. La paille et la poutre." in Economica. L'ad hoc en économie. La paille et la poutre. A. AUTUME, CARTELLIER, J., Paris: 267-290.

BENETTI, C., CARTELIER, J., (1987). "Monnaie, valeur et propriété privée." Revue Economique $38 \mathrm{~N}^{\circ}$ 6: 1157-1170.

BOHANNAN, P. (1959). "The impact of money on an African subsistence economy." The Journal of Economic History Volume 19(Issue 4): 491-503.

BOYER-XAMBEAU, M. T., DELEPLACE, G. et GILLARD, L. (1986). Monnaie Privée et Pouvoir des Princes. Paris, CNRS, Presses de la Fondation Nationale des Sciences Politiques.

BRAUDEL, F., LABROUSSE, E., (1977). La Méditerranée. L'espace et les hommes. Paris, Arts et métiers graphiques.

BRUNNER, K., MELTZER, A.H., (1971). "The use of money : money in the theory of an exchange economy." American Economic Review: 784-804.

CHAPMAN, A. (1980). "Barter as a universal mode of exchange." L'homme, revue française d'anthropologie. 20, $\mathrm{N}^{\circ} 3$ : 33-84.

CLOWER, R. W. (1967). "A reconsideration of the microfundations of monetary theory." Western Economic Journal 6: 1-8.

CLOWER, R. W. (1995). "On the Origin of Monetary Theory." Economic Inquiry 23: 525-536.

DICTIONNAIRE (2003). "Le Grand dictionnaire français-latin (15931628): histoire, types et méthodes". Secondary "Le Grand dictionnaire françois-latin (1593-1628): histoire, types et méthodes", http://tactweb2.chass.utoronto.ca/cgi-

bin/tactxml/tactxml2.pl/rendico/5nic_ah?PFC103900\#here.

GODBOUT, J. (1992). L'esprit du Don. Paris, La Découverte.

GODELIER, M. (1996). L'énigme du Don. Paris, Fayard.

JEVONS, W. S. (1876). Money and the Mechanism of Exchange. New York, D. Appleton and Company.

JEVONS, W. S. (1957). The Theory of Political Economy. New York, Kelley \& Millman.

JONES, R. A. (1976). "The Origin and Development of Media of Exchange." Journal of Political Economy 84 (4): 757-775. 
KIYOTAKI, N., WRIGHT, R., (1989). "On money as a Medium of Exchange." Journal of Political Economy 97 n² 4: 927-954.

KIYOTAKI, N., WRIGHT, R., (1991). "A contribution to the pure theory of money"." Journal of Economic Theory 53 (2): 215-235.

KNAPP, G. F. (1973). The state theory of money. New York, Sentry Press.

MAUSS, M. (2002). "Essai sur le Don. Forme et raison de l'échange dans les sociétés archaïques." Article originalement publié dans l'Année de la Sociologie, seconde série, 1923-1924: 106.

OH, S. (1989). "A Theory of Generally Acceptable Medium of Exchange and Barter." Journal of Monetary Economics. 23 (1): 101119.

ORLEAN, A. (1991). "L'origine de la Monnaie." Revue du MAUSS 14: 126-152.

ORLEAN, A. (2003). "Réflexion sur les fondements institutionnels de l'objectivité marchande." Cahiers d'économie politique 44: 181-196.

OSTROY, J., STARR, RM., (1974). "Money and Decentralisation of Exchange." Econometrica 42(6): 1093-1114.

OSTROY, J., STARR, RM., (1990). "The transaction role of money". in Elsevier Sciences Publishers. The transaction role of money. B. M. FREIDMAN, HAHN, F.H., 1.

POLANY, K. (1968). Primitive, Archaic, and Modern Economies: Essays of Karl Polanyi by George Dalton. Boston, Beacon Press.

POLANYI, K. (1996). La Grande Transformation. Paris, Gallimard.

SAHLINS, M. (1976). Age de pierre, âge d'abondance, l'économie des sociétés primitives. Paris, Gallimard.

SERVET, J.-M., THERET, B., YILDIRIM, Z., (2007). "Universalité du fait monétaire et pluralité des monnaies, de la confrontation coloniale à la rencontre des sciences sociales". in Odile Jacob. Universalité du fait monétaire et pluralité des monnaies, de la confrontation coloniale à la rencontre des sciences sociales. Paris.

STARR, R. M. (1972). "Exchange in Barter and Monetary Economies." Quarterly Journal of Economics LXXXVI: 290-302.

WILLIAMSON, S., WRIGHT, R., (1994). "Barter and Monetary Exchange Under Private Information." The American Economic Review 84 (1): 104-123.

184 Economie et Institutions $-\mathrm{n}^{\circ} 10$ et $11-1$ er $\& 2$ e semestre 2007 